\title{
Purinergic signalling in the inner ear-perspectives and progress
}

\author{
Gary Housley • Jonathan Gale
}

Published online: 14 July 2010

(C) Springer Science+Business Media B.V. 2010

\section{Editorial}

This special issue of Purinergic Signalling brings together ten peer-reviewed papers that consider complementary aspects of the wide-ranging involvement of extracellular nucleotide and nucleoside signalling in the inner ear. The credit for this initiative sits with the Editor, Professor Geoffrey Burnstock, who reflected upon the depth of knowledge of the involvement of ATP and adenosine on the operation of the hearing organ and provided this opportunity to show-case current research in this area and its implications for auditory and vestibular neuroscience. As guest editors, we greatly appreciate the efforts that the contributing authors have extended to communicate their expertise and enthusiasm for the field to colleagues and other kindred spirits interested in the broad context of purinergic signalling at the molecular, cellular and systems levels. We also thank the production staff at Springer for their support and advice in the organisation of this special issue. The papers provide reviews and perspectives, along with original research contributions, that define the breadth of the purinergic

G. Housley $(\bowtie)$

Translational Neuroscience Facility and Department of Physiology, School of Medical Sciences, University of New South Wales, Sydney, NSW 2052, Australia

e-mail: g.housley@unsw.edu.au

J. Gale

UCL Ear Institute,

332 Gray's Inn Road,

WC1X 8EE London, UK

J. Gale

Department of Cell \& Developmental Biology,

332 Gray's Inn Road,

WC1X 8EE London, UK signalling complex and consider how the elements influence the development, physiology, pathophysiology and ageing of the cochlea and vestibular end-organs. The studies also show how the base of knowledge of purinergic signalling in the cochlea enables translational research with considerable promise for treatment of hearing loss.

The issue opens with consideration of the influence of extracellular ATP signalling on cochlear ontogeny. The paper by Tritsch et al. describes the phenomenon of pulsatile ATP release in Kölliker's organ and its role in the spatiotemporal entraining of auditory neurotransmission that commences in pre-hearing rodents just after birth. In the developing cochlea, Kölliker's organ is a transient epithelium that is part of the greater epithelial ridge and is formed of inner supporting cells that have also been called Kölliker's cells or inner sulcus cells. These cells are both adjacent to and medial to the primary sound encoding cells, the inner hair cells. The paper reviews the recent discovery that ATP released in bursts by the Kölliker's cells diffuses to the adjacent inner hair cells, and via P2Y (G proteincoupled receptors) and P2X receptors (ATP-gated ion channels), causes depolarization that if sufficiently large causes $\mathrm{Ca}^{2+}$-spiking leading to release of vesicles of glutamate neurotransmitter. The distal neurites of the type I spiral ganglion neurons that innervate the inner hair cells are depolarized and these primary auditory neurons fire action potentials that propagate along the axon from the cell somata to the cochlear nucleus in the brain stem. The establishment of periodicity of firing in the auditory nerve fibres as the synaptic connections are made at the hair cells and cochlear nucleus are fundamental to the establishment of auditory transmission and processing of auditory signals. One interesting feature of this purinergic response is the changes in cell shape that are observed in those Kölliker's cells. The current paper provides further insight into the 
purinergic signalling complex associated with this activity. These cells appear to integrate ATP release, ATP receptor activation and downstream second messenger signalling via $\mathrm{Ca}^{2+}$ and inositol trisphosphate $\left(\mathrm{IP}_{3}\right)$ into an activity that spreads as a wave from a single Kölliker's cell effecting changes in intracellular $\mathrm{Ca}^{2+}$ and cell shape. Similar synchronous coupling between supporting cells is also known to occur in outer supporting cells. In the latter cells, as for Kölliker's organ, ATP signalling is implicated in communication of tissue differentiation in the last stages of cochlear ontogeny. However, beyond the onset of hearing (from the second post-natal week when Kölliker's organ is reduced down to a layer of inner sulcus cells), cell stress and damage may engage downstream signal cascades important in the response to injury. The second paper, by Majumder et al. firstly provides a concise introduction to the cochlea and a review of gap-junction function in the cochlea. Secondly, it considers the outer supporting cell region and provides new data on the mechanism for connexin hemi-channel mediated ATP release and the process of extracellular signal propagation via ATPmediated activation of ATP receptors linked to the ATP release mechanism. The paper by Lahne and Gale is complementary to these studies in considering mechanisms of intercellular communication and the associated downstream signalling partners. It reviews the evidence for purinergic receptor-mediated injury responses and investigates damage-induced signalling in the cochlear explants in greater detail. Two independent intercellular $\mathrm{Ca}^{2+}$ waves are described; both depend upon purinergic activation but differing in their sources of $\mathrm{Ca}^{2+}$, indicating $\mathrm{P} 2 \mathrm{X}$ dependence versus P2Y-dependence. Thus, the waves propagate through different cell types on the basis of expressing different purinergic receptor profiles. Thus the same damage-stimulus can be signalled to both hair cells and supporting cells via different receptor mechanisms.

In the mature cochlea, purinergic signalling is broadly distributed in the cochlear epithelium, including not just the principal elements of hair cells and spiral ganglion neurons, but all the epithelial cells lining the cochlear partition (of the endolymphatic compartment). Thus P1 (adenosine receptors) and $\mathrm{P} 2 \mathrm{X}$ and $\mathrm{P} 2 \mathrm{Y}$ receptors are also expressed by the supporting cells of the organ of Corti and various epithelial cells lining the cochlear partition. EctoNTPDases and ecto-nucleoside diphosphokinase are co-localised with these purinergic signal transduction elements, often to the level of subcellular specific polarisation. Isoforms of these ecto-enzymes often appear matched to modify nucleotide hydrolysis and conversion to marry with the substrate specificity of associated purinergic receptors. The paper by Ito and Dulon summarises the knowledge of purinergic receptor expression in the cochlea and vestibular endorgans and examines the complexity of $\mathrm{P} 2 \mathrm{Y}$ receptor signal coupling, particularly with regard to the spiral ganglion neurons. Here, ATP may act as a neurotransmitter or neuromodulator alongside other neurochemicals, particularly glutamate and acetylcholine, via G protein-coupled receptors, and engage a common metabotropic effector, a putative TRPC non-selective cation channel. Thus, the firing properties of the primary afferent neurons within the inner ear are likely regulated by $\mathrm{P} 2 \mathrm{Y}$ receptor/TRPC ion channel-induced depolarization and $\mathrm{Ca}^{2+}$ entry. Developing this theme of cross-talk between purinergic receptors, the paper by Harada reviews the evidence for the interaction between nitric oxide signalling and ATP receptor-mediated $\mathrm{Ca}^{2+}$ signalling by the spiral ganglion neurons and the inner and outer hair cells. Nitric oxide (NO) acts chiefly through cGMP-dependent protein kinase to regulate $\mathrm{Ca}^{2+}$ homeostasis. In the cochlea, production of NO via nitric oxide synthase (NOS) in-turn involves a $\mathrm{Ca}^{2+}$ feedback mechanism mediated by ATP receptors.

Ion homeostasis is integral to the physiology of hearing. The endolymphatic compartment (scala media) of the cochlea has an ionic composition more akin to the cytoplasm than to extracellular plasma or cerebral spinal fluid. This high $\mathrm{K}^{+}$, low $\mathrm{Na}^{+}$and $\mathrm{Ca}^{2+}$ milieu reflects the primary role of this chamber as a reservoir for the transducer current that passes through the sound transduction ion channels on the tips of the sensory hair cells. The driving force for this sound transduction process is provided by a positive endocochlear potential developed by the stria vascularis tissue in the lateral wall of the cochlea. Thus, $\mathrm{K}^{+}$is excreted into scala tympani, passes through the apical membrane of the hair cells as the transducer current, exits via the basolateral $\mathrm{K}^{+}$channels of these cells, is then moved from the Corti-lymph region through the gap-junction coupled supporting cells and returned to the $\mathrm{Na}^{+} / \mathrm{K}^{+}$ATP pumps in the lateral wall. Under stressor conditions such as loud sound or ischemia, ATP is released from the cells of the cochlear partition, possibly via connexin and pannexin hemichannels but also by cell damage, and activates $\mathrm{P} 2 \mathrm{X}$ and $\mathrm{P} 2 \mathrm{Y}$ receptors in a paracrine fashion. In the case of $\mathrm{P} 2 \mathrm{X}$ receptors, ATP release activates these non-selective ion channels and provides a shunt of $\mathrm{K}^{+}$out of scala media, short-circuiting the cochlear battery in a process that reflects a humoral hearing adaptation mechanism. In the paper by Zhu and Zhao, the permeability of ATP-activated ion currents to $\mathrm{K}^{+}$is explored in isolated cochlear supporting cells. The authors discuss the potential for enhanced $\mathrm{K}^{+}$siphoning through $\mathrm{P} 2 \mathrm{X}$ receptors when extracellular $\mathrm{K}^{+}$is increased in the cochlea under conditions such as loud noise. Parallel to the roles of P2X receptors in the cochlea, P2Y receptor expression is equally prominent. The paper by Huang et al. provides a comprehensive characterization of $\mathrm{P} 2 \mathrm{Y}$ receptor expression in the cochlea during development. The 
data implicate P2Y receptors in the differentiation of the cochlear epithelium, and describes expression of $\mathrm{P}_{2} \mathrm{Y}_{4}$ receptors in the Kölliker's organ, correlating in time with the purinergic signalling complex involved in pulsatile ATP release. Expansion of $\mathrm{P}_{2} \mathrm{Y}_{4}$ receptor expression in the stria vascularis around the onset of hearing also occurs which in this case is correlated with the known inhibitory feedback of $\mathrm{K}^{+}$secretion via PKC-inhibition of $\mathrm{KCNE} 1 \mathrm{~K}^{+}$channel subunits in the marginal cells which are part of the $\mathrm{K}^{+}$entry path from the lateral wall recirculation pathway. In contrast, the hair cells are shown to have highly compartmentalised and diverse $\mathrm{P} 2 \mathrm{Y}$ receptor localization, with $\mathrm{P}_{2} \mathrm{Y}_{2}$ and $\mathrm{P} 2 \mathrm{Y}_{6}$ polarised to the apical (sensory) pole, while in the cochlear outer hair cells, $\mathrm{P} 2 \mathrm{Y}_{1}$ protein lines the lateral wall. The latter is the site for prestin-mediated outer hair cell electromotility, providing the cellular basis for the mammalian "cochlear amplifier" that confers unparalleled sensitivity and frequency selectivity to the mammalian cochlea. Whether $\mathrm{P}_{2} \mathrm{Y}_{1}$ receptors are involved in the modulation of prestin remains to be seen.

The final three papers develop the translational aspect of purinergic signalling with regard to hearing loss. The paper by O'Keeffe et al. considers the expression and regulation of elements of the purinergic signalling complex in the cochlea under normal conditions and with noise exposure. This study shows regulation of mRNA expression and protein expression of the UDP-associated ectonucleotidases (NTPDase 5 and 6) and $\mathrm{P} 2 \mathrm{Y}$ receptors $\mathrm{P}_{2} \mathrm{Y}_{6}, \mathrm{P} 2 \mathrm{Y}_{14}$. In particular, the expression of NTPDase 6 within the inner hair cell is striking and further investigation into the cellspecificity and function of this isoform is warranted. The paper by Telang et al. considers the effects of ageing on cochlear function and on $\mathrm{P} 2 \mathrm{X}_{2}$ receptor expression in a mouse model. Immunocytochemical detection indicates that the expression of $\mathrm{P} 2 \mathrm{X}_{2}$ receptors is increased in the organ of Corti and spiral limbus after noise exposure in young but not older animals. It is possible that this reflects a reduced ability of the cochlea to provide protective adaptation to sound stress and may contribute to increased susceptibility to noise-induced hearing loss with age. The final paper, by Vlajkovic et al., provides an exciting first indication of the translational potential of the field. Here, evidence is provided that the $\mathrm{A} 1$ receptor agonist adenosine amine congener is an effective treatment for noise-induced hearing loss, particularly when treatment was started $6 \mathrm{~h}$ (rather than $24 \mathrm{~h}$ ) after noise exposure. These data, alongside studies of adenosine receptor expression in the cochlea, support the hypothesis that adenosine acts via A1 receptors to ameliorate noise-induced hearing loss. The mechanism of action is not entirely clear, but it most likely results from a reduction in the reactive oxygen and nitrogen species that may trigger hair cell death.

In summary, we hope that the breadth of investigations into cochlear purinergic signalling described herein indicate the importance of this system in the inner ear. Further support for research in this area is clearly warranted and is likely to provide therapeutic agents, targets and treatments for hearing and vestibular disorders. 